\title{
Analysis of the FX traders' work using the Naturalistic Decision Making framework
}

\author{
Bill Davilas \\ Nicolas Marmaras \\ National Technical University of Athens \\ Department of Mechanical Engineering \\ Sector of Industrial Management Operations Research \\ Production Management Laboratory - Ergonomics Unit \\ Email address: bdavilas@,central.ntua.gr_ Email address: marmaras@,central.ntua.gr
}

\begin{abstract}
Motivation - The present study deals with an aspect of the Foreign Exchange traders' work, the choice to trade directly with other traders or indirectly through electronic brokers. Research approach - Based on data obtained through systematic observations, and adopting the naturalistic decision making framework, we searched to identify persisting patterns of the context within which the execution of orders has been carried out. Findings/Design -The results show that direct trading is preferred within contexts different from those of indirect trading. Originality/Value - Apart from the confirmation of the central hypothesis of the NDM according to which experienced decision makers prefer the pattern matching than the analytic evaluation of alternatives, these findings have also implications for both the theory of FX trading and the design of decision support tools for this complex cognitive task.
\end{abstract}

Keywords

Complex Cognitive task, Foreign Exchange Market, trading behavior, Naturalistic Decision Making

\section{INTRODUCTION}

The introduction of information technology tools in decision making domains is inevitably accompanied by the need for increased formalization (Nathanael \& Marmaras, 2008). By increased formalization we mean rearrangement of work practices towards more standard and official procedures. This augmented formalisation may constrain the decision makers to effectively respond to the particularities of the situation at hand, and consequently may lead to the entire or partial abandonment of the tools.

In the present paper, we investigate this phenomenon in the domain of Foreign Exchange (FX) traders. Traditionally, FX traders negotiated and executed the orders directly with other FX traders or brokers, via phone or telex. In 1992 electronic broking systems have been introduced in the domain, rendering the trading more "automatic" and indirect. Despite the advantages of the indirect trading, the traditional direct trading has not been abandoned and is still used by the traders. Adopting the naturalistic decision making paradigm, we try to identify in which contexts the traders prefer the direct than the indirect mode of trading.

\section{A FEW WORDS ABOUT FX TRADING}

The FX market (both in its retail and the wholesale-interbank mode) was first founded as a result of changes in the international monetary system, passing from the fixed exchange rate requirements of Breton Woods till 1970s' to the flexible rates of today. The elimination of national government controls, the freedom for transnational transactions and advances in technology, have all led to an enormous amount of foreign currencies exchanged on a daily basis (\$1.9 trillion bought and sold globally, compared to $\$ 1.6$ billion a day on the New York Stock Exchange) (Bjonnes et al., 2003). One of the main characteristics of the market is the 24-hour activity and the geographical dispersion. Other important characteristics of that market are (Bjonnes et al., 2003):

- low transparency because of decentralization,

- low execution cost of trades (owed to deal automation) and

- increased speed of trade execution.

FX traders are employed by Commercial and Investment Banks and work in the Dealing Rooms at the Banks' Treasury Divisions. They deal with purchasing and delivering foreign exchange either on a proprietary or on customers' order fulfillment basis. Their main goal is to make profits by selling currency at a currency rate greater than that of purchase, or-conversely- by buying currency at a currency rate lower than the expected selling currency rate. As the traders may 
choose the time at which they will carry out a particular trading, they have to predict future currency rates and trends. Predictions are made based on a multitude of information such as the currency rates movements, changes of national and international economic indices (e.g. unemployment rates, gross domestic product, gross national products, inflation rate), political and economic events, other traders' opinions and market sentiments.

A FX trader can give quotes (acting as a market maker) when other traders directly ask him/her for quotes (i.e. the FX trader receives an incoming trade). Alternatively s/he can ask other traders for buy-sell quotes (outgoing trades); the other traders are in this case acting as market makers while the trader asking for quotes is now named "market taker" (Bjonnes et al., 2003). These trading options belong to the direct trading category made via telephone or electronic communication networks, since the auction is bilateral. However, traders can also give quotes or enter orders to Electronic Broking Systems or physical brokers, therefore relying on indirect trading; brokers, either electronic or physical, manage all buy-sell orders and quotes submitted by traders and match them according to currency rate, time of entry and quantity criteria.

From a FX market taker point of view, the spread is defined as the difference between the "buy" currency rate (ask which is always higher than the selling currency rate) and the "selling" currency rate (bid) (Bjonnes et al., op.cit). Conversely seen, a market maker gives always-higher selling currency rates than buying quotes. From a theoretical FX Market Structure point of view, a market taker, who has just bought (as a market taker) a currency and wants to sell it later on (as a market taker again), usually waits till the bid currency rate reaches the ask currency rate of the initial trade, in order to experience neither profits nor losses (break even point) (Bjonnes et al., op.cit.).

FX traders deploy a set of tools in analyzing and explaining market movements. FX traders frequently face unpredicted situations: in such cases s/he usually tries to make sense of "where the market goes" in order to predict exchange movements. Fundamental Analysis and Technical Analysis tools help towards this end. Fundamental Analysis consists of analysis and prediction methods based on macroeconomic and microeconomic models. Their main contribution is the integration of various national and international economic indices into consistent models of analysis with predictive capabilities of the real value of currencies. On the contrary, Technical Analysis tools focus on the analysis of past prices and chart interpretation (chartism): a large part of practitioners' community think of technical analysis tools as a means for future trend and turning points prediction.

These two categories of tools are not mutually exclusive in use, but are consulted on a complementary basis, although predictions are not fully reliable (Curcio et al. 1997). However, FX theorists come to the general conclusion that the shorter the time horizon of trading is (i.e. the higher the frequency of trades is), the more the reliance on technical analysis tools is, because the integration of fundamentals into an analysis or prediction formula presupposes plenty of time and exhaustive analytical process; however time pressure, especially in high frequency-intra day trading, is one of the main task-setting factors (Lui et al. 1998).

As far as the nature of the trade itself, we identify 2 generic categories: FX traders make market orders when they want to execute a trade (buy/sell) of a specific amount of a currency at the best current/available rate. Conversely, they set limit orders to execute when they want to execute a trade (buy/sell) of a specific amount of money at a determined currency rate (which is determined either on a proprietary basis or is set by the customer).

We can conclude that FX traders perform a complex decision making task (Marmaras \& Pavard 2000). In fact decisions are taken within and for a complex system consisting of many interrelated and interacting components -i.e. the other FX traders- and factors -i.e. the factors determining the economic environment-; there is uncertainty regarding the time at which one or more events occur, and the severity of changes they cause to the system; there are multiple quantitative objectives to be achieved, often conflicting (e.g. increasing profits versus minimizing losses risk).

\section{The introduction of computerized trading}

Foreign Exchange traders, till 1970, could negotiate and execute the orders directly, via the phone or telex. Alternatively, broking companies were taking the responsibility to intermediate between a "buying" and a "selling" party, announcing currency rates for execution, and after executing the trade, those companies were also responsible for trade settlement.

In 1992 the electronic broking systems were introduced. Using them FX traders can execute an order in the following way: they either input a "price" (the rate at which they want to execute the order) and the quantity of money they want to exchange, or they just enter a quantity of money for exchange stating that it is a market order (an order that they want to be executed for the given quantity at the best available price/rate). An electronic broker acts as an order "warehouse": different orders are matched with time and requested price (rate) priority. When the rate of orders being executed via an electronic broker is close t the rate requested by the FX trader, the rate input field in the interface begins to flash (as an indication that the order is all about to execute). Submitting an order is anonymous and counter party details are revealed only after the trade is executed.

Furthermore, the electronic offer additional opportunities: 
- FX traders can have access to chart display that presents real time information about currency pairs (fully flexible in display mode).

- FX traders can also access market information using special applications, that usually present national and international economic indices, news, interest rates, Gross National and Domestic Product and incorporate current market activity (such as last trades all over the world, biggest trades etc.).

- Finally, FX traders can use fundamental and technical analysis tools via specific formulas use and visualization facilities.

"Reuters" electronic broker and "E.B.S." (Electronic Broking Systems) are the most favorite electronic broking systems used in the Inter bank market nowadays. "E.B.S." is the outcome of major market making banks partnership, as a response to "Reuters" high shares in electronic trading.

However traders still use direct trading in executing trades. The introduction of computerized trading (electronic broking) did not eliminate direct trading volumes; on the contrary, it seems that electronic broking triggered the evolution of direct trading. Indeed, "Reuters" made the next step in direct trading and auctioning during "90s with the introduction of bilateral electronic communication systems. These systems-networks resemble to the traditional telephone system that was formerly used, serving as a tool for typing conversations (with up to 4 other traders). Traders soon rendered them the leading tool for direct bilateral trading since they could substitute voice bargaining for typing written auction.

FX theorists generally agree that electronic broking systems have undoubtedly led to higher market centralization by representing (Bjonnes et al., 2003):

- best available currency rates (either best currency rates available in the whole market or best currency rates currently available by the system, only from credit approved banks),

- own trader's deals-trades,

- the direction of all trades executed by the system - characterized either as given (at the bid currency rate-buy) or paid (at the ask currency rate-sell),

- group market data (currency rates and directions for all trades, and not only for those that voice brokers can announce) leading to higher post trade transparency (transparency after trade execution: data announcement such as buy/sell, currency rate, counter parties details).

In which cases FX traders execute trades directly or indirectly according to the literature

As far as the FX traders' choice between direct and indirect trading is concerned, we should first state that direct trading permits anonymity vis-à-vis the market, since the trades executed directly are neither displayed on a globally accessed screen nor publicly announced. On the contrary, in indirect trading, the counter parties' identities are disclosed after trade execution, when the two counter parties get the details of each other and the trade is displayed on the electronic broker screen. Moreover, FX traders, when trading directly think that they have the discretion to accept or reject currency rates given by a counter party, however daily practice and related literature show that each time a FX trader receives an incoming quote query "has" to respond, by giving quotes to the initiator of the contact. On the other hand, participation in indirect trading is not obligatory, as FX trader fully decides on when to place on order or the currency rate $\mathrm{s}$ /he wants to trade at. In addition, not only literature in this field but our ethnographic observation data also revealed that FX traders give 2 way quotes (when another trader asks for currency rates) in direct trading; on the contrary, FX traders in indirect trade give only the direction of the trade (buy/sell) and currency rate.

FX theorists such as Bjonnes et al. (2003) agree that FX traders prefer direct trading for making large trades: it is a common sense among practitioners of the field that when a FX trader asks for a large trade, there is a lot of information revealed (such as the personal expectancies of the trader asking for the trade or possible private information). As a consequence, practitioners claim that direct trading is preferred since it does not permit information on large trades to be disclosed. Furthermore, FX trading behavior research (Bjonnes et al. 2003, Bjonnes 2003) has come to the conclusion that in periods of distress (e.g. after shocks in the market, an announcement or an unexpected event or a catastrophe), FX traders rely more on indirect than direct trading. Researchers state that, when FX traders act in such an environment, they think of electronic brokers as the safest way to trade: the danger to deal with a well informed FX trader theoretically splits to all of the electronic broking system's current users (while in direct trading FX traders have to address this issue individually).

\section{Why FX trading is an appropriate application for Naturalistic Decision Making?}

Recent discoveries of trading \& investing decision processes' studies as well as recent empirical studies' evidence (Olsen 2000, Olsen 2002) suggest that classical decision making methods "may be of limited value" for financial decision makers (Olsen, 2002). Classical decision paradigms describe the decision making process within a perfect information and given preferences environment, which is deterministic in nature and always "function with a knowable set of natural laws that are independent of time and context" (Olsen, 2002).Taking subjective expected Utility Theory 
paradigm as a key driver of research, those scientific methods promise precise prediction results and desired outcomes' accomplishment. However, the aforementioned methods may not apply in the FX \& Investment decision making environments, mainly because:

1. Financial decision makers seem that are not interested in selecting optimal solutions, but usually prefer feasible solutions,

2. the greater cognitive effort and higher costs of classical decision making may make less accurate and less costly rules of thumb more attractive (Olsen, 2002).

Generally speaking, financial decision making processes are context dependent: in contrast with well structured decision environments where decision makers rely heavily on quantitative analysis and rules, ill structured and complex decisions (such as deciding on whether to sell or buy a currency and when) seem that are based more on intuition inducing procedures (mainly characterized with satisficing) (Olsen, 2002).

Naturalistic Decision Making focuses on the way decision makers recognize and respond to indeterminate decision situations (Olsen, 2002). Describing a naturalistic Decision Making situation, researchers of the field (Olsen, 2002 \& Klein, 1997) recognize the following task-setting factors:

1. experts' extensive domain-specific knowledge and experience (Olsen, 2002)

2. complex, ill-defined decision making context,

3. use of sizing up / situation awareness tactics on behalf of financial decision makers, instead of applying context free optimization rules (Olsen, 2002).

Schemata, patterns and mental models, facilitating memory and experience recall as well as improving generalization process, seem that are deployed by decision makers in cases of incomplete and ambiguous information, time stress and novel situations.

As already stated in the introduction, in the present paper we search to identify the context factors that contribute to the choice of trading execution channel that traders make in their everyday activity, adopting a different research paradigm than that usually used by the researchers of the domain: the Naturalistic Decision Making paradigm. We have been mainly driven in our research by the fact that naturalistic decision paradigm has not been heavily applied to the financial decision making process. We will hopefully add to the effort of (i) putting greater emphasis on "clinical" training of trading rookies instead of studying textbooks in a context free class environment (Olsen, 2002) and (ii) the design of decision support tools for this complex cognitive task.

\section{METHOD}

In order to acquire the required data, we used the ethnographic approach. During a period of three months, we carried out systematic observations of the work of an experienced FX trader specialised in Euro/US Dollars transactions, at a Dealing Room of a major Greek commercial bank. We tried to trace any information perceived by the trader as well as every observable activity performed by him. When possible, explanatory questions regarding his actions were posed to the trader. Furthermore, we also traced intraday Euro/US Dollars (EUR/USD) data for the specific period (restricted by the fact that we had limited time available for trades' recording, due to the Bank's policy; we did not have permission to stay in the area for the whole working time and we had to leave the FX desk at about 13.00-13.30); these data concern the open currency rate, the close currency rate, the minimum/maximum currency rate, and the transactions volume, within a 10-minute time interval between records.

For the needs of the present study, we focused on the analysis of data regarding the execution of customers' orders given to the FX trader by the Back Office or other desks of the Dealing Room. Aiming to identify eventual differences in the contexts in which the trader chooses to trade an order directly with another trader or indirectly through the electronic platform, at a first phase we determined two broad factors that characterize the trading context. The first one is the state of the market which can be described by two parameters: (i) the total amount of money spent on buys-sells at the specific moment in the whole FX market (or volume of trades), and (ii) the current level of the EUR/USD rate (which was the currency pair that was traded in the $95 \%$ of recorded cases). The second factor is the order to be executed that can be described by four parameters: (i) the quantity of money requested for buy/sell, (ii) the requested currency rate, (iii) the time of delivery (or valeur) and (iv) the time that order request was submitted.

At a second phase we searched to visualize the different contexts in which the orders have been executed. To do that we determined the following three-grade scales for each of the six parameters of the context:

- for the volume of trades: low for 1-400 million EUR/USD trades, medium for 400-700 million EUR/USD trades, high for 700-1000 million EUR/USD trades; 


\section{Davilas $\cdot$ Marmaras \\ Analysis of FX traders' Work Using the N.D.M. Framework}

- for the current rate: falling when the EUR/USD rate was decreasing for the 10 last minutes, stable when the rate was more or less stable for the10 last minutes, and rising when the EUR/USD rate was increasing for the 10 last minutes;

- for the quantity of money requested for buy/sell: low for 1-4 million EUR/USD, medium for 4-7 million EUR/USD, high for 7-10 million EUR/USD;

- for the requested currency rate: under, within or above the open-close range of the rate for the specific 10minute time interval;

- for the time of delivery (or valeur): no valeur, delivery on the same day, or delivery on the next day.

- Last, we decided to depict on the same graph the direct or indirect execution of each trade.

Using these three-grade scales for these six context parameters, we were able to draw a visual representation of the context for each trade performed during the period we observed the FX trader. Figure 1 presents two examples of contexts within which a direct and an indirect trade have been performed.

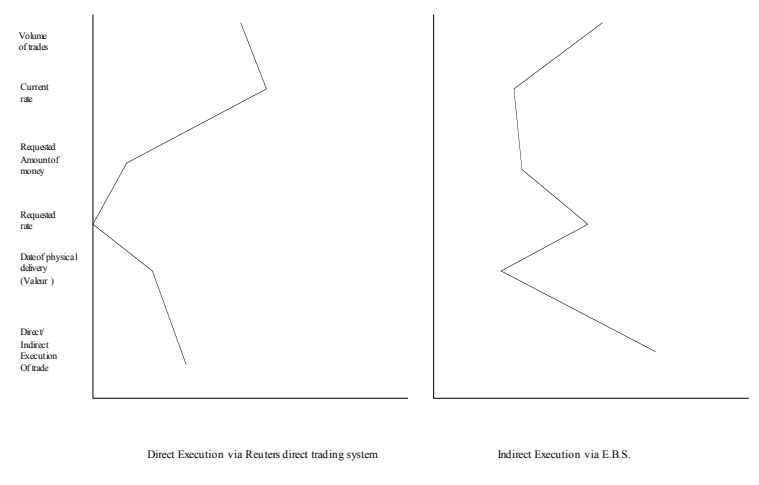

Figure 1: Two examples of contexts within which a direct and an indirect trade have been performed

At a third phase, observing the visual representations of the context for the twenty four (24) cases of order execution trades we searched for persisting context patterns which would differentiate the cases of direct and indirect trading.

\section{RESULTS}

As already stated, during the period of our systematic observations, there were twenty four (24) orders executed by the trader. Nine of them were executed directly (via the Reuters direct trading system), while the remaining (15) were executed indirectly, via electronic brokers (E.B.S. or Reuters). We identified three (3) persisting context patterns for the direct trading cases, and four (4) for the indirect trading cases, while the remaining of these orders do not qualify into any of the context patterns mentioned above.

More specifically, the persisting context patterns within which the trader chose the direct trading are the following:

Pattern d1: medium volume of trades, flat current EUR/USD rate, low requested amount of money, no specified requested rate (market order), without specified date of delivery (Figure 2-left side, three trades).

Pattern d2: high volume of trades, flat to slightly rising current rate, medium amount of money requested, requested rate lying within the current EUR/USD range, without a specified date of delivery (Figure 2-centre, three trades).

Pattern d3: minimum volume of trades, marginally falling current market rate, very low requested amount of money, requested rate above current market levels, without specified date of physical delivery (Figure 2-right side, three trades). 

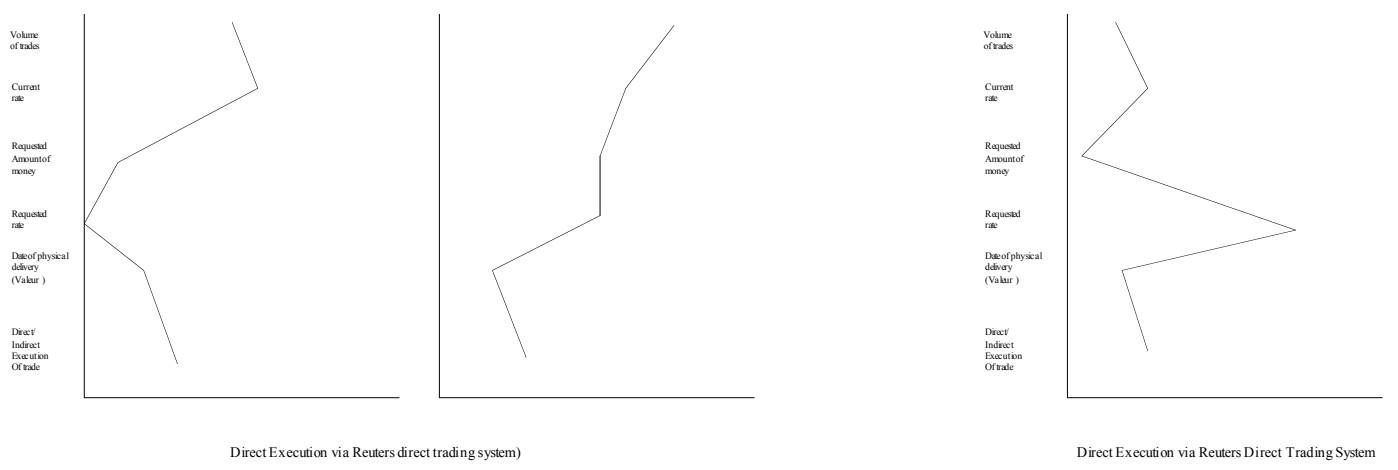

Figure 2- Direct execution of trades

As far as the persisting context patterns within which the trader chose the indirect trading, they are the following:

Pattern ind1: medium volume of trades, falling current rate, low requested amount of money, requested rate within market levels, without specified date of delivery (Figure 3-left side, two trades).

Pattern ind2: medium volume of trades, falling current rate, low requested amount of money, requested rate within the current EUR/USD rate, without specified date of deliver (Figure 3-left to center side, three trades).

Pattern ind3: medium volume of trades, flat current rate, low requested amount of money, requested rate lying below the current EUR/USD range of rate, without a specified date of delivery (Figure 3-right to center side, three trades).

Pattern ind4: medium volume of trades, flat to slightly rising current EUR/USD rate, low requested amount of money, requested rate lying below the current EUR/USD range, specified date of delivery the following day of the execution date (Figure 3-right side, two trades).
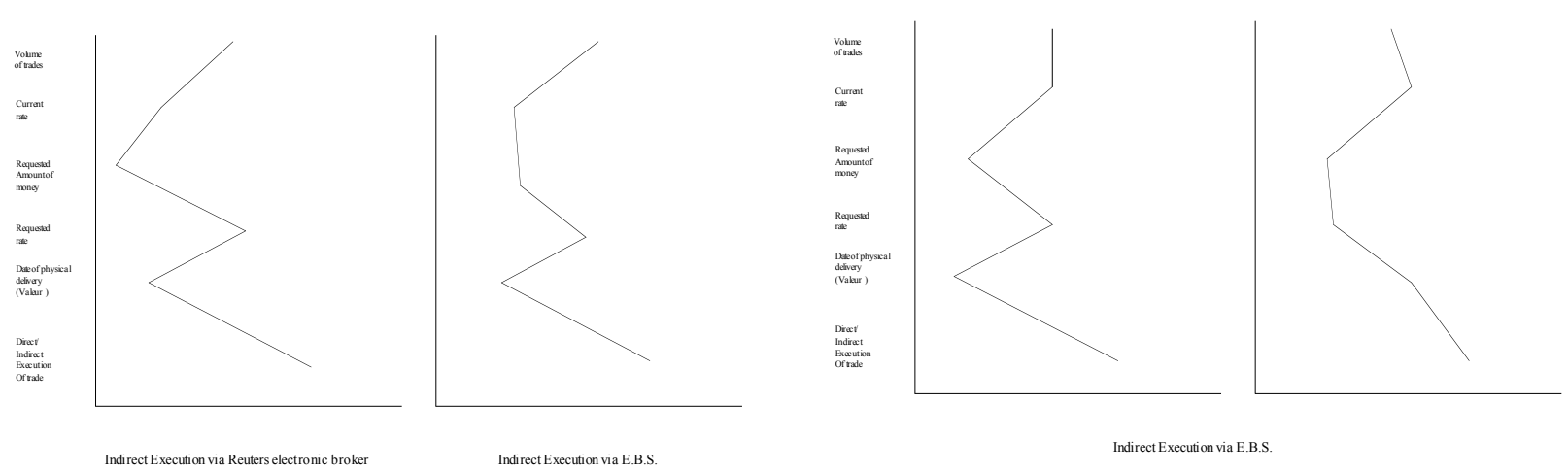

Figure 3- Indirect execution of trades

Therefore we can conclude that the contexts within which the trader chose to execute an order directly are different to those he chose to trade indirectly. The differences of the contexts form distinctive patterns of parameters values.

\section{CONCLUSIONS}

The results presented above are partial and preliminary. In fact they do not concern the whole FX traders' task but a sub-task, and were obtained by studying only an experienced trader. However a number of conclusions can be drawn with both a generic value for the paradigm of naturalistic decision making (NDM), as well as a specific value for the study of traders' work aiming to support it. 
A central hypothesis of NDM is that proficient decision makers use situation-action matching decision rules with the basic structure of "do A because it is appropriate for situation S", rather than concurrent choice process with the structure of "do A because it has superior outcomes to its alternatives". Matching differs from concurrent choice in three respects. (i) Options are evaluated sequentially one at a time. Evidence exists that even when presented with several options, decision makers quickly screen most of them by comparing them against a standard, rather than with one another, and then focus on one, or at most two, options, which are compared (Beach, 1993; Montgomery, 1988). (ii) Options are selected or rejected based on their compatibility with the situation (Endsley, 1997; Klein, 1998; Pennington, \& Hastie, 1993), or the decision maker's values (Beach, 1990), rather than on their relative merits. (iii) The process of matching may be analytic but more often it relies on pattern matching and informal reasoning (Cohen, Freeman, \& Wolf, 1996; Klein, 1998; Lipshitz, 1993; Pennington , \& Hastie, 1986).

The results of our study confirm this hypothesis. In fact as we have shown the choice to trade an order directly or indirectly is based on the recognition by the trader of type of situation at hand, i.e. by pattern matching, and not by an analytic evaluation of the two options for each case of trading.

A second conclusion that can be drawn from the present study concerns the fertility of the knowledge that can be gained adopting the NDM paradigm. In fact, as already mentioned, previous studies dealing with the choice of direct or indirect trading and using classic analytical approaches, have find only one relevant factor, this of the volume of the amount to be traded (Bjonnes et al., 2003). In contrast, the results of our study shows that there are much more factors that describe the situations under which a FX trader may choose one or the other type of trading. This finding, if confirmed by studying other FX traders, will enrich the theory of trading and may have positive practical implications both on the traders' education as well as on the design of decision support tools. Specifically, an information technology system equipped with a user interface representing the information related to the state of the market as well as to the parameters of order to be executed in a graphical way similar to the patterns recognized by the traders, is expected to provide a valuable support to the traders' decision making process.

\section{REFERENCES}

Bank of England, (2000). The Foreign Exchange Market. Fact Sheet

Beunza, D., Stark, D., (2004). Ecologies of Value in a Wall Street Trading Room. U.P.F. Working Paper No. 735

Bjonnes, G. H., Rime D., (2003). Dealer Behavior and Trading Systems in Foreign Exchange Markets. Working Paper, Norges Bank, Research Department, Oslo, Norway

Rime et al., D., (2003). New Electronic Trading Systems in Foreign Exchange Markets. Norges Bank and Stockholm Institute for Financial Research

Curcio, R. (1997). Do Technical Trading Rules Generate Profits? Conclusions from the Intra-Day Foreign Exchange Market, International Journal of Financial Economics, Vol. 2, pp.267-280

Evans, Martin D.D. (1998). “The Microstructure of Foreign Exchange Dynamics” mimeo, Georgetown University.

Klein, G. (1997). Naturalistic Decision Making: Where are we going? In Caroline E. Zsambok \& Gary Klein (Eds.) Naturalistic Decision Making, Lawrence Erlbaum Associates, pp.383-397

Knorr Cetina, K., Bruegger. U, (2002). Traders' Engagement with Markets, A Post social Relationship, Theory, Culture \& Society, Vol. $19(5 / 6)$, pp.161-185

Lui, Y.H., Mole, D. (1998). The use of fundamental and technical analyses by foreign exchange dealers: Hong Kong evidence, Journal of International Money and Finance, Vol. 17, pp.535-545

Marmaras, N., Nathanael, D. (2005). Cognitive Engineering Practice: melting theory into reality. Theoretical Issues in Cognitive Science, 6(2), pp.109-127

Marmaras, N., Pavard, B., (2000). Problem driven approach for the design of information systems supporting complex cognitive tasks. Cognition, Technology \& Work, 1(4), pp.222-236

Olsen, R. (2000). "The instinctive mind on Wall Street: Evolution and Investment Decision Making." Journal of Investing, Vol.9, No. 4, pp.7-14

Olsen, R. (2002). "Professional Investors as Naturalistic Decision Makers: Evidence and Market Implications". The Journal of Psychology and Financial Markets, Vol. 3, No. 3, pp. 161-167 
Zsambok, C. (1997). Naturalistic Decision Making: Where Are We Now? , In Caroline E. Zsambok \& Gary Klein (Eds.) Naturalistic Decision Making, Lawrence Erlbaum Associates, pp.3-16 